\title{
RAPID PROTOTYPING USING LOCAL AREA NETWORK (LAN) TECHNOLOGIES
}

David J. Roberts

\section{Naval Ocean Systems Center \\ San Diego, California}

\begin{abstract}
An ethernet local area network and ISO/OSI transport and network layer software package was used to establish connectivity between functional components of an intergatewaying testbed. Standard interfaces were used to support session functions, thus, simplifying the coding effort on the variety of testbed hardware and insuring the portability of developed code. An overall thrust of the rapid prototyping effort was the minimizing of prototype specific code and efficiency modularization of software elements.
\end{abstract}

\section{INTRODUCTION}

The following are the results and philosophical approach employed in a Navy sponsored research effort at the Naval Ocean Systems Center (NAVOCEANSYSCEN), San Diego. The specific project developing the technology is the Unified Networking Technology (UNT) program, a 6.2 block program out of the Office of Naval Technology (ONT), Washington, D.C.

Because of the complexity and technological risks associated with the project, both discrete simulations and rapid prototyping were used to finalize detailed design issues. While the use of discrete simulation, Simscript II.5 (CACI), is an accepted technique for verifying hardware and software designs, the rapid prototyping technique will be the underlying theme of this paper.

Details with regard to data types, frames lengths, and specific system data has not been included in this presentation because of security considerations. Any persons or agencies seeking such information may apply through appropriate security channels to the NAVOCEANSYSCEN, San Diego. The inter-service and agency sharing of information has always been encouraged at NAVOCEANSYSCEN and will be supported on a need to know basis.

\section{BACKGROUND}

The stated goal of the UNT project was to provide more robust communications for existing and future multimedia intership systems. General goals include: rapid reconfiguration of communication links, enhanced jamming sensing and recovery, load balancing and shedding, and extension of communications link geographic ranges. It should be pointed out that the goals of the UNT program only addressed the interplatform connectivity issues while a sister program was concerned with intra-ship databases and systems.

Typically Naval communication systems have, in the past, used point to point interface designs that optimize features of a single communication circuit. A variety of valid rationales do exist for this design technique, and will not be explored within context of this paper. Because of the years of development and varying functionality of communication systems, the interface of any two systems may not be a trivial task. To interface dissimilar communications networks or source data bases electrical, flow control, data frames, and other compatibility issues must be resolved. This has normally resulted in the production of a Requirements Interface Documents which addresses, in detail, hardware and protocol specifications. Such documents are generally manhour intensive and applicable to only those systems under consideration. Generalization to a wider communication scheme is rarely possible. Figure 1. illustrates a typical point to point connectivity within a shipboard environment. Although the maximum number of connectivities could be expressed as the number of systems squared, rarely are all systems interconnected. Rather, some system will exchange information only with others of a similar functional nature. Where functional necessities dictate, requirement interface specifications are generated on a one to one basis. However, the emphasis of the UNT program is not intraplatform data source communication, but rather interplatform communication links - an area of little interoperability.

The focus of the UNT program is an intergatewaying problem of how to successfully carry varying message types over a wide range of possible circuit types. In the case of one communication either lacking capacity or being jammed, an alternative circuit can be selected based on the priority of the message and the probability of link success.

At the onset of the UNT architectural design effort the complexities of internetting dissimilar systems was realized. Further, the focal point of UNT being interconnection of the physical communication circuits presented a massive and previously unexplored area of research. Interfaces on a point to point nature did exist for shipboard data systems, but little work had been done in actually intergatewaying intership communications circuits. Thus, the UNT project was left with two decisions: to attempt the effort using traditional interface

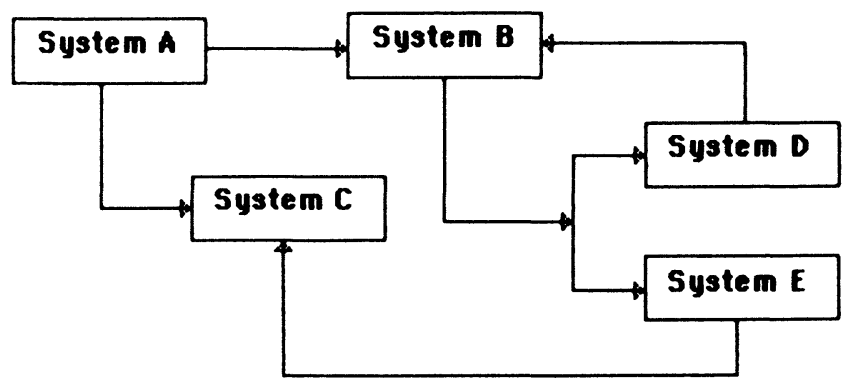

Figure 1. Typical shipboard data systems interfaces 
techniques, or to seek an alternative technology that could be used to merge existing and future fleet systems, while still reducing the scale of effort.

A departure from the traditional point to point interfacing techniques was decided because of the physical number of circuit interfaces and, more importantly, because of the constant evolution of Navy communication systems (i.e. new modems, cryptos).

A combination of modern Local Area Network (LAN) and the imposition of a virtual format was selected as a technique that satisfied interface requirements. Another benefit is that it allowed separation of tasks into controllable and economically feasible units. The details of this technique and the UNT system architecture will be the subject of the following sections.

\section{HARDWARE CONFIGURATION}

The hardware configuration of the demonstration testbed was based upon very strict functional and physical requirements. Existing and future communication systems placed many constraints on the testbed design. Additionally COMSEC considerations provided further restrictions.

As an eventual goal the testbed was designed to emulate a multi-tasking multi-processor environment based on an initial analysis of the volume of traffic to be handled by the system and the required redundancy of such communications equipment.

To minimize development time, off-the-shelf hardware was selected for the laboratory testbed. IBM-AT class microcomputers were selected for emulation of I/O level communications circuit controllers (Link Controllers), simple incoming and outgoing routers, and a message generator, while a more sophisticated SUN III system (System Administrator) collected data on system performance, message reception, and presented the results in a graphic format for the system user. Individual processor components were interfaced through use of ethernet boards in a standard ethernet configuration.

UNT Link Controllers (LC) handled three types of information flow: message data, status with regard to receipt of messages, and status with regard to the link condition.

The specific functions of the LC were:

a. Formats incoming data into a virtual frames for MC/LC communications

b. Formats outgoing data into the link specific format

c. Setup links and nodes (maintains link specific routing table)

d. Multiple access protocols for MC communications

e. Reliable delivery of data blocks

f. Output link status such as traffic loading or jamming to the MC

g. Data forwarding when node acts as relay

h. Encryption/decryption device interface

The Multinetwork Controller (MC) shown in Figure 2. has been presented as three functionally separate processors interfaced to the LCs via a high speed bus and to the origination data sources by either existing interfaces or a Network Interface Unit under development by the Distributed Command System (DCS) Program (a sister program at NAVOCEANSYSCEN)

Specific Multinetwork controller function are:

a. Monitor the performance of LCs to include traffic flow, signal quality, unusual signals present, etc.

b. Analyze channel data for failure/congestion prediction

c. Utilize an expert system which contains typical link characteristics and propagation prediction data

\section{d. Analyze data for flow/congestion control}

e. Analyze data for data link selection

f. Maintain a dynamically updated routing table of all links and end user nodes

g. Control of data flow to the originating communication system by providing appropriate

h. Prioritizing of data, voice, and graphics information in a multimedia modem

While the UNT testbed used "canned" data (i.e. NTDS tape input) the eventual goals will be to interface directly to the shipboard Local Area Network (LAN) under development by the Distributed Command System (DCS) program at NAVOCEANSYSCEN. Such a Network Interface Unit would conform to DCS specifications (TCP/IP with a user defined ISO/OSI Presentation Layer') and would allow the multinetwork controller to connect in a transparent matter to the DCS LAN.

No attempt was made in the laboratory testbed to consider tempesting requirements since preliminary work used sanitized data and work with live data was performed in vaulted areas. A functional layout of the system is presented in Figure 2.

Using LAN technology to interface system components provided two main advantages: simplification of timing schemes between processes and compartmentalization of software development. The LAN, because of its bus speed and CSMA-CD ${ }^{2}$ algorithms eliminated much of the detailed work with state diagrams and bus level interrupt or polling schemes. The LAN provided a "near transparent" path between processes. The compartmentalizing of the software effort will be the subject of the following section.

\section{SOFTWARE DEVELOPMENT}

With the elimination of much of the low level interface software efforts could be concentrated on the higher level application and session management. The ISO/OSI model provided the functional guidelines and was strictly followed throughout the coding effort. Although most of the communications circuits considered by UNT did not make full utilization of the ISO/OSI layering technique, enough compatibility did exist to make the ISO/OSI model a practical technique for software development and future design considerations. Within UNT components off-the-shelf software was used to maintain connectivity in the ethernet environment. FUSION ${ }^{3}$, a software product from Network Research Corporation, was used to support ISO/OSI Network and Transport layers. 


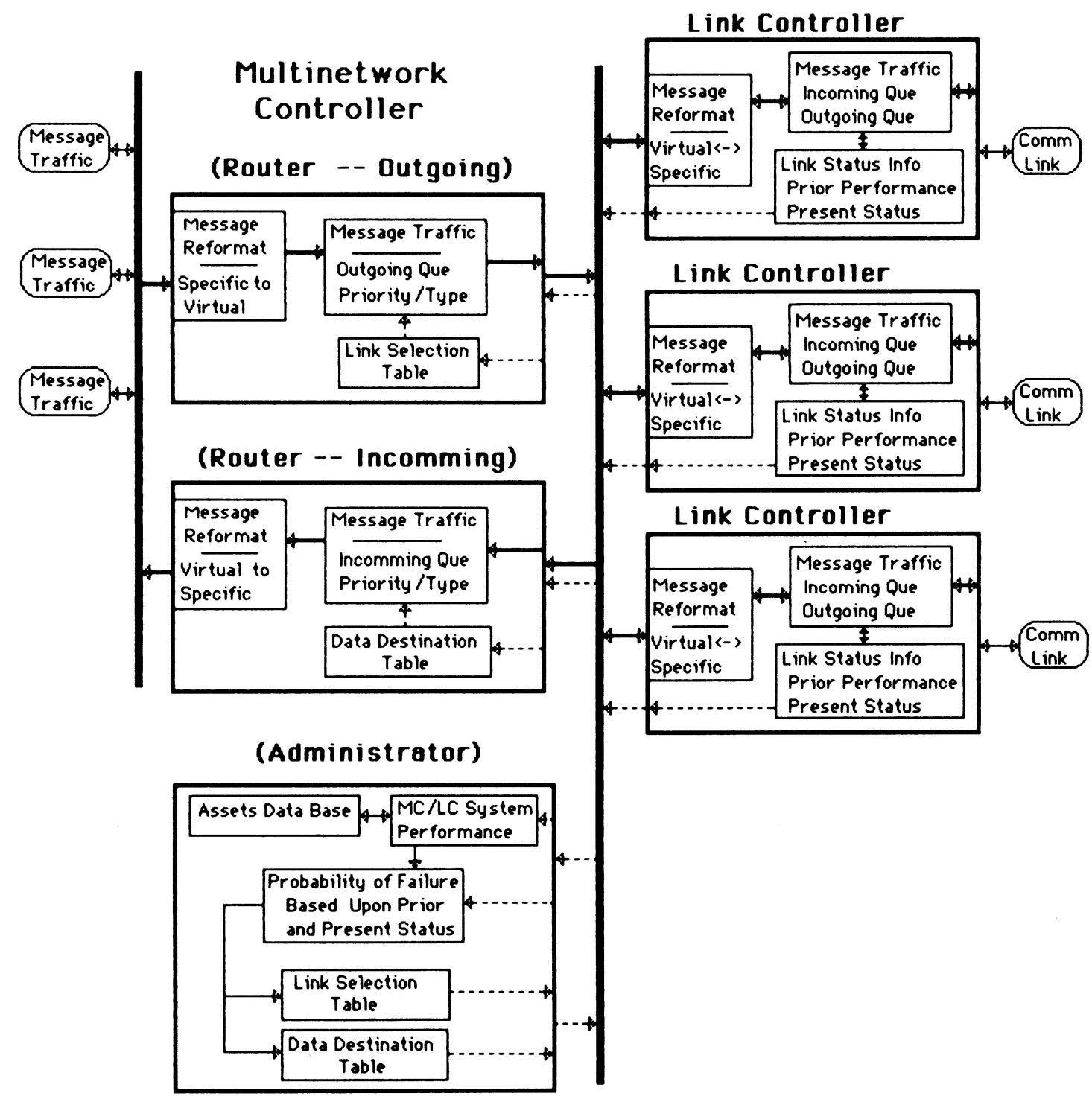

Figure 2. Functional Layout of the UNT Testbed

Although the product was not optimally efficient over a "custom" ethernet interface, it was deemed satisfactory since it did not degrade the overall system performance and required little original development effort. Throughput bottlenecks (i.e. low speed communication links) had been identified early in the system design and the ethernet backbone was never viewed as an area of concern. Rather, the ethernet LAN was seen as a transparent media for interprocess communications as shown in Figure 3.

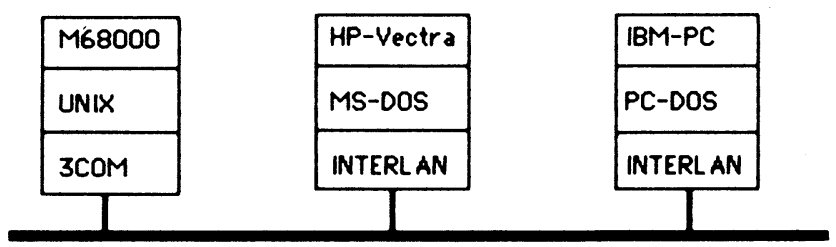

Figure 3. UNT Testbed Connectivities
Physical and Data Link layers were handled by the ethernet equipment and device drivers with the FUSION software interfacing to several vendors data link devices offering both portability between devices and operating systems offered, and standardization of interface for the upper level ISO/OSI layers (see Figure 4). The FUSION network socket manager provided a logical interface between the host and communication oriented layers. This standardization in the testbed was considered a major point for both the development of prototype software and transitioning to a field test system.

\section{EXPECTED PAYOFF}

The UNT will develop algorithms that may be incorporated in many existing and planned systems. Algorithms will support rapidly reconfigurable networks, optimum radio selections (HF, UHF or EHF), multimedia ordered delivery (voice, data, graphics), optimum message routing and reliable message delivery. Candidate systems for transition are: Link 11, HFAJ, C2P, UHF SATCOM DAMA, NAVCOMPARS, JTIDS \& NESP. 


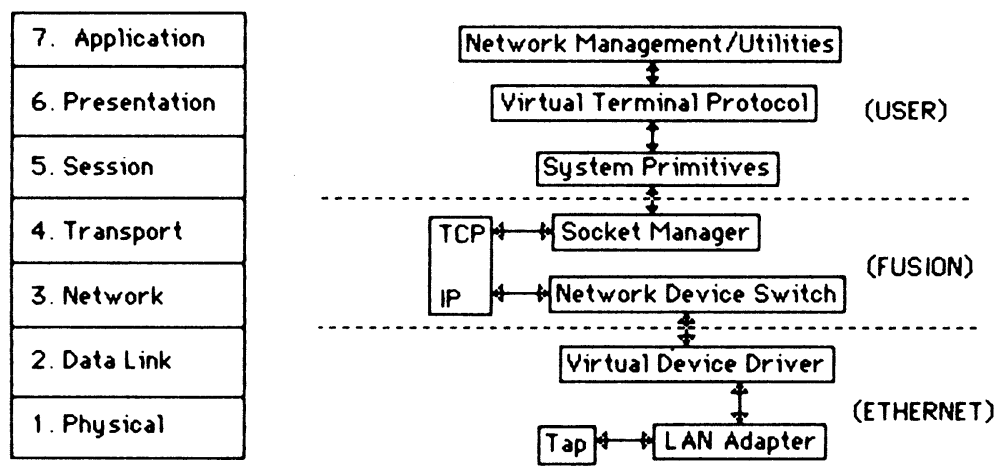

Figure 4. The UNT Testbed Software with Reference to the ISO/OSI Model

\section{APPLICABILITY OF UNT TECHNOLOGY}

With questions of interoperability of communications systems a key issue within and between the Services, the technology being developed by UNT should be of interest to a wide audience of potential users. Additionally, the "practical" development mode of UNT could provide simple baselines for more wide-sweeping systems. The use of a LAN to simplify the software development effort makes sense from the viewpoint of placing the emphasis of the effort on the development of higher OSI/ISO levels of software, rather than lower level, prototype specific code.

As stated previously in the paper, the NAVOCEANSYSCEN, San Diego would be pleased to cooperate with both industry and other governmental agencies in the development intergatewaying technologies.

\section{REFERENCES}

1. Black, Uyless D. (1983), Data Communications, Networks, and Distributed Processing, Reston Publishing Company, Inc., Reston, Virginia.

2. Martin, James (1981), Computer Networks and Distributed Processing, Prentice-Hall, Inc. Englewood, New Jersey.

3. Network Research Corporation (1986), Fusion Version 3 Product Fact Sheet, Oxnard, California.

ETHERNET is a trademark of Xerox Corporation FUSION is a licensed trademark of Network Research Corporation

HP-Vectra is a registered trademark of the Hewlett Packard Corporation

IBM is a registered trademark of International Business Machines Corporation

IBM-AT is a trademark of IBM Corporation

IBM-PC is a trademark of IBM Corporation

MS-DOS is a trademark of Microsoft Corporation

PC-DOS is a trademark of IBM Corporation

Simscript II.5 is a trademark of CACI Corporation

SUN III is a trademark of Sun Microsystems, Inc.

UNIX is a trademark of Bell Laboratories 